\title{
Estrategias y metodologías de formación de equipos interdisciplinarios para trabajar en hábitat
}

\author{
Interdisciplinary teams training strategies and \\ methodology to work in habitat
}

\section{Elena Lucca*}

Fecha de recepción: 25-04-2017 - Fecha de aceptación: 16-09-2017

Hábitat y Sociedad (ISSN 2173-125X), n..$^{\circ} 10$, noviembre de 2017, pp. 15-34.

http://dx.doi.org/10.12795/HabitatySociedad.2017.i10.02

\begin{abstract}
Interdisciplinary group work is not a spontaneous construction because it has an epistemological rupture with the way the uni-disciplinary knowledge is acquired, introducing common vital dimensional and nonreferential action. To teach interdisciplinary capacities is a procedure we developed at university. We build these capacities by introducing some characteristics for the formation of the initial groups; this fits within the theory of the operatives groups. This kind of groups needs specific roles of coordination and facilitation, in order to go through the process toward interdisciplinary. In a second instance, when the interdisciplinary team take this proposal to the territory, we define the habitat and methodology to meet the inhabitants. The encounter professionals and inhabitants empower the action in a decision making position about the characteristics they would like to have for their own territory. These methodologies and practice made the team and inhabitant assume the fundamental learning to take charge of their own territory and habitat.
\end{abstract}

\section{Key words}

Interdisciplinary, Coordination, Sustainability, Habitat

\begin{abstract}
Resumen
La interdisciplina es una construcción a la que no se accede espontáneamente ya que, en sí, conlleva una ruptura epistemológica con la forma de adquirir los conocimientos unidisciplinarios, e introduce una dimensión vital, grupal y no solo profesionalmente referencial al involucrarse en la acción. Formar la capacidad interdisciplinaria en la universidad es una opción que desarrollamos aquí. Se plantean algunas características de la formación inicial de equipos, encuadrada en la teoría de los grupos operativos, asistidos por una coordinación que facilita el proceso hacia la interdisciplina. Proceso que requiere de una lectura y elaboración de las líneas de poder que se juegan en el interior de esos grupos y en las formas de transferencia hacia la intervención en las comunidades. Al llevar la propuesta al territorio, definimos hábitat y metodologías para el encuentro con pobladores. Encuentro que coloca a esos pobladores en el lugar de decisión de las características que desean para el territorio habitado por ellos. Tanto la formación en gabinete como la práctica en terreno son instancias de aprendizaje fundamental para actuar en el hábitat.
\end{abstract}

\section{Pallabras clave}

Interdisciplina; Coordinación; Sustentabilidad; Hábitat

\footnotetext{
* Doctora en Geografía, Profesora Universidad Nacional del Nordeste. Saavedra 2632, Resistencia, Chaco 3500, Argentina. C.e.: elenalucca2@hotmail.com.
} 


\section{Introducción}

Abrir la posibilidad de un trabajo conjunto, profesionales diversos y habitantes de un lugar, es el desafío. Y que el grupo de profesionales actúe desde un punto de vista común, elaborado a través de un proceso interdisciplinario, es lo deseable. Intervención unificada que implica saberes, análisis y acciones consensuadas, como es necesario que sean las propuestas socioambientales territoriales en el hábitat. Un camino que va más allá de las acciones que proponen las estructuras políticas, más allá de una mirada unidimensional profesional, que nos involucra como profesionales y ciudadanos del territorio donde vivimos y actuamos y con los habitantes del mismo.

La universidad puede ser el lugar apropiado para capacitarse y comenzar a pensar interdisciplinariamente. Y para el proceso de formación necesario proponemos crear espacios donde pueda practicarse la interdisciplina. Espacios que, por lo general, se dejan al azar, ya que no es habitual en las carreras universitarias unidisciplinarias sumar diversos enfoques disciplinares. Espacios donde se forme la capacidad de estructurar un pensamiento común, tomar decisiones más allá de la propia disciplina y actuar partiendo de la creación conjunta. Espacios curriculares que vayan creando mentes hacia una mirada total de las situaciones y que a su vez exijan profundizar en las propias disciplinas para aportar una mirada integrada a ese caldero común que son nuestros asentamientos, ciudades o espacios habitados.

La propuesta es delinear lo que es un proceso interdisciplinario, luego dar algunas pautas para la formación en espacios determinados específicamente para capacitar en interdisciplina y finalmente hacer algunas consideraciones no exhaustivas para la actuación en terreno de los equipos interdisciplinarios.

Los conceptos desarrollados en el segundo punto (La construcción de la interdisciplina en el ámbito universitario) provienen, mayormente, de la práctica realizada durante 15 años en Extensión Universitaria de la Universidad Nacional del Nordeste Argentino (UNNE). Esta práctica, registrada metódicamente en cuadernos de bitácora de cada grupo-equipo coordinados, ha sido analizada con la teoría de la sistematización, los esquemas de observación y los indicadores que corresponden a la comunicación digital y analógica, indicadores explícitos e implícitos, en la evolución de esos grupos-equipos conformados por estudiantes y profesionales de distintos campos, y con aplicación posterior a distintas situaciones del hábitat territorial e institucional.

El esquema de trabajo de estos grupos-equipos está basado en los grupos T, o training groups, en la teoría de la información, teoría de los sistemas, teoría de la comunicación, la comunicación no verbal, la cibernética, la autoorganización, los laboratorios sociales de sensibilización y la teoría de la creatividad.

En el tercer punto (Hábitat, equipos interdisciplinarios y habitantes de un territorio) vamos a terreno, definiendo previamente hábitat, y desarrollamos algunas conceptualizaciones y procedimientos que pueden emplearse en la bajada del grupo interdisciplinario al territorio, en el encuentro con los pobladores y en la inclusión de la mirada sobre los procesos ambientales del lugar. Estos conceptos y procedimientos han sido elaborados a partir de planes y proyectos en terreno que trabajamos desde distintas instituciones y que han requerido también de la confluencia de miradas y saberes.

Comenzamos con algunas definiciones para acordar un enfoque. 


\section{Interdisciplina, poder y sustentabilidad}

Cuando hablamos de interdisciplina, estamos hablando de hecho de una intervención o construcción frente a un tema o situación donde se requiere una respuesta o propuesta unificada, más allá de la mirada desde un solo ángulo disciplinar.

La INTERDISCIPLINARIEDAD se funda en una epistemología construccionista, ya que no solo son necesarias las miradas confluyentes sobre el objeto, sino aún más, es necesario el fuego que cocine, que permita transformar esa comida en algo diferente a la suma de sus ingredientes. Esa transformación de las miradas unidisciplinarias a través de ese FUEGO-PROCESO para la construcción de una mirada única sobre el objeto de interés, es esencial para el abordaje de los temas y sistemas complejos (Lucca, 2014, p. 15).

Y todas las situaciones en que nos enfrentamos en el hábitat poseen componentes complejos, inabordables desde un solo punto de vista. $\mathrm{Al}$ encararlas unidisciplinariamente estamos escotomizando la respuesta a las mismas, por lo tanto, empobreciéndolas o despojándolas de esa complejidad o riqueza que cada situación conlleva. Tampoco, frente a la complejidad, es posible una aproximación de varias disciplinas yuxtapuestas, una sumatoria de distintas miradas, ya que complica la respuesta sin asumir su complejidad.

En la Carta de Transdisciplinariedad, ${ }^{1}$ Primer Congreso de la Transdisciplinariedad (1994, art. 4), se expresa “... la transdisciplinariedad reside en la unificación semántica y operativa de las acepciones a través y más allá de las disciplinas". Estos dos puntos, la unificación semántica y operativa, son el objeto de la formación interdisciplinaria, que en su práctica puede arribar a momentos privilegiados transdisciplinarios.

Es necesario tener en cuenta que cada situación de la realidad que se nos presenta a resolver implica una totalidad. Para captar esta totalidad de una situación es necesaria una elaboración, proceso y cocinado común entre las personas que intervienen, que da como resultado un enfoque único.

Esta categoría de totalidad de las situaciones complejas del hábitat implica un dominio omnilateral y dominante del todo que es "mucho más" que las partes y más que la visualización de alguna parte en sí. Es decir, el comportamiento de estas situaciones implica un sistema complejo de interrelaciones, decodificable solamente desde la situación como un todo.

Esto nos lleva a definir interdisciplina de la siguiente manera:

Es una forma de conocer para la acción, un sistema no lineal, complejo, que implica la captación de la totalidad de un tema/situación, lo que lleva a una modificación de valores y una nueva pauta cultural cambiando las formas o parámetros del conocer de cada una de las disciplinas intervinientes, ya que conjuga y destila distintos enfoques que, al mezclarse, producen una visión/acción de un nivel más completo, adecuado y profundo.

En esta definición que proponemos aparece enunciado lo que llamo quiebre epistemológico disciplinar, al modificar los parámetros de conocer de las disciplinas. Este quiebre provoca un momento que requiere especial consideración, ya que, frente al mismo y la pérdida de seguridad que conlleva, los miembros adoptan distintas formas de enfrentarlo. De las cuales puede surgir un intento de hacerse cargo de la tarea
1 Consideramos el término transdisciplinariedad equivalente a interdisciplina en este caso, por su sentido de uso en el documento que se cita. 
del equipo proponiendo distintas soluciones y generando un acopio de poder hacia alguna de las disciplinas o un modelo de funcionamiento sin jerarquías, fusional, que permite el control de esa tarea por parte de algún miembro o disciplina. Por el contrario, el camino hacia la interdisciplina requiere que el flujo de saberes sea abierto y sujeto a una permanente incertidumbre que deje espacio a la introducción de nuevas relaciones complejas y a las respuestas vitales que parten de la "intuición, del imaginario, de la sensibilidad y del cuerpo en la transmisión de los conocimientos" (ob. cit., art. 11).

Las situaciones del hábitat a las que nos enfrentamos y que requieren intervención, muestran su complejidad al comenzar su análisis. ¿Por qué, entonces, por lo general, son encaradas desde una lógica unidisciplinaria?

El conocimiento profesional establecido es un sistema de poder. La interdisciplina es una herramienta para encontrar la fisura en ese poder. Herramienta que nos permite una aproximación objetiva mediante el cocinado de las interpretaciones subjetivas que implican: CONCERTACIÓN y PARTICIPACIÓN (Lucca, 2014, pp. 14-15).

La interdisciplina aplica la metodología de la intersubjetividad objetiva construyendo desde esta metodología un pasaje que, aunque no enfrenta los flujos de poder del saber disciplinario, da posibilidad de un tejido para empezar a crear un campo común.

Tomamos como ejemplo un trabajo interdisciplinario realizado para la concreción de un Plan de Manejo Sustentable de los Espacios Naturales en la Trama Urbana de la ciudad de Resistencia, Chaco, Argentina. Este plan se aborda con un equipo de profesionales universitarios convocados por asociaciones ambientalistas en convenio con la Municipalidad de la ciudad, compuesto por las siguientes áreas: aspectos históricos, hídricos, forestales, ecosistémicos, uso de los espacios, gestión y gobernabilidad, sociales, culturales y educativos.

Después del análisis inicial por área, se definen dos perfiles: el ámbito natural en sí y el ámbito de la interacción, uso y gestión de esos espacios naturales. Al comparar estos perfiles a una situación de los espacios meta o ideal, obtenemos los indicadores de estado y de direccionalidad de las acciones que se pueden proponer. En esta elaboración aparecen los temas interdisciplinarios que requieren propuestas interaccionales conjuntas de las disciplinas que componen el equipo.

Mientras en el transcurrir de las primeras reuniones el grupo comparte desde cada área sus datos, a partir de la aparición de los perfiles a trabajar comienza un intercambio de contenidos y prioridades a tomar en cuenta, lo cual va creando el pensamiento común que desemboca en las decisiones interdisciplinarias.

El hábitat es complejo y entre sus complejidades existe la necesidad imperiosa de que sea sustentable (Peredo Parada y Aedo Zúñiga, 2016). Si estamos interviniendo en él, la transferencia que hacemos al intervenir puede ser creadora de dependencia o de sustentabilidad. Aclaramos, cuando hablamos de sustentabilidad, lo hacemos desde la definición ecológica y de la termodinámica, que plantean la sustentabilidad como la "capacidad de carga" de los territorios, es decir, la capacidad de sustento de la vida en un determinado espacio territorial, de acuerdo con sus elementos naturales existentes (Rieznik Lamana y Hernández Aja, 2005).

¿Qué significa crear sustentabilidad en el hábitat? 
La constatación,

- primero, que la tierra es un sistema abierto en energía, pero cerrado en materia, y

- segundo, que es más fácil convertir los materiales de la corteza terrestre en energía, que la energía en materia,

- ello hace que la gestión de la materia sea el principal tema de la sustentabilidad.

Cuando actuamos en el hábitat estamos manipulando materia.

Nos interesa aquí remarcar desde qué concepción usamos la palabra sustentabilidad en este documento. Basándonos en las ciencias biológicas, que acuñaron este término, decimos que sustentabilidad proviene de reconocer, aceptar y mantener el sustrato existente. Lo proponen las ciencias ecológicas en relación con la capacidad de carga, de un territorio determinado, para mantener la evolución propia de ese espacio con sus componentes y necesidades.

La sustentabilidad fuerte es aquella que se basa en la lógica de lo viviente y busca la perpetuidad de los elementos y la cobertura natural de la que depende la especie humana en su totalidad (Carreño et al., 2003; Lucca, 2016). La implicancia de esta concepción significa darle prioridad a ese medio natural en el que nos asentamos. Cualquiera sea la intervención que propongamos sobre el hábitat, sería oportuno encararla desde este enfoque, es decir, analizando y mirando el territorio natural y lo que él nos presenta con sus mecanismos de autoorganización propios. Enfoque que significa revisar el estado de los espacios naturales y proponer su mantenimiento o recuperación, pensando e insertando lo construido o a construir para la habitabilidad humana, en convivencia con esos espacios. Estamos, así, direccionando hacia la sustentabilidad fuerte posible, al pensar y actuar acorde a la complejidad ecosistémica de ese hábitat.

Ya no planeamos solamente desde el ángulo humano nuestra instalación, sino que desplazamos el eje hacia el espacio territorial, cuidando de interferir lo menos posible en los procesos que allí se producen. Pasamos así de la centralización en lo humano hacia lo que el territorio nos indica como apto para convivir con él.

Actualmente muchas formas de intervención en hábitat se plantean desde la lógica económica o social, sin tomar en cuenta el territorio, lo cual provoca problemas que luego hay que enfrentar con grandes costos económicos y sociales. La propuesta acá es, entonces, desplazar el poder y colocarlo en lo territorial, en primera instancia.

Y para realizar esta modificación, que implica un cambio en la concepción de como intervenir, la propuesta del trabajo interdisciplinario es una vía y herramienta fundamental de elaboración. Ya que interdisciplina, en su esencia misma, significa un manejo de poder distribuido entre los distintos aspectos que hacen a una acción, distribución de poder y equilibrio, y una posibilidad de intercambio y transferencia.

\section{La construcción de la interdisciplina en el ámbito universitario}

En general las universidades poseen la necesidad y algunos ámbitos estructurados y determinados para el abordaje, la aplicación y práctica en los temas que se presentan en la vida cotidiana de sus lugares de de- 
sarrollo. Organismos dentro del mismo campus o en la sociedad que les rodea.

Por ejemplo, los departamentos de Extensión Universitaria son espacios que se vuelcan a las comunidades a través de propuestas o proyectos donde se ponen en marcha mecanismos de aplicación profesional. Sería deseable que estos proyectos o propuestas sean trabajadas desde la interdisciplina. La consigna podría ser: CREAR ESPACIOS INTERDISCIPLINARIOS DE PRÁCTICA, con resultados valorados. Construir así, desde la base de la formación profesional, la mentalidad interdisciplinaria.

Poner en marcha esta propuesta nos lleva a ampliar la formación universitaria unidisciplinaria con miras a aplicar nuestros conocimientos en la práctica profesional misma. La universidad puede ser el ámbito por excelencia de esta formación para la práctica.

Y reiteramos, no es espontáneamente que se accede a la interdisciplina. Hay momentos claves de resistencia que es importante aprender a superar y traspasar hasta lograr una propuesta abierta y flexible. La formación en interdisciplina y en los roles específicos de coordinación/facilitación de los equipos requiere de un proceso pedagógico y didáctico que estructure una forma diferenciada del hacer individual. Por ejemplo, que un profesional se ubique en una "distancia operativa" con respecto a un grupo o equipo, para permitirle superar sus etapas conflictivas, casi siempre referidas a la preservación individual o a la supremacía de un enfoque profesional particular, requiere de un proceso formativo pautado y formulado a partir de la práctica en situaciones emergentes. Lo mismo que pertenecer como miembro a un equipo interdisciplinario. Ambos lugares requieren de elaboración teórica y ejercitación práctica o supervisión en terreno.

Para estructurar una formación, comenzamos teniendo en cuenta algunos puntos y consideraciones básicas y generales.

- La presentación de un tema/objeto/sujeto/situación de atención interdisciplinaria. Es importante aclarar que los temas del hábitat humano, que involucran los del hábitat de las otras especies, es materia de un enfoque interdisciplinario sin ningún reparo. Ya sea que aparezca el tema a elaborar desde algún campo específico como arquitectura, geografía, ciencias agrarias, ciencias sociales, biología, etc., el tema del hábitat requiere siempre del diagnóstico y manejo interdisciplinario.

- Inicialmente, los roles específicos son asumidos por la persona docente a cargo de la formación interdisciplinaria.

- La conformación del equipo que va a elaborar una propuesta en terreno puede tener un miembro en función de dirección, que en el momento de la elaboración interdisciplinaria va a participar como un integrante más. Lo que significa que, en el momento del proceso formativo, su estatus es equivalente al de los otros miembros, depositando en coordinación la función de regular esa elaboración. Como es probable que este miembro haya citado a los otros participantes que van a componer el equipo, coordinación aclara la ubicación equivalente de todos los miembros, con el propósito de volcar hacia la tarea de elaboración conjunta. Posteriormente, en caso de asumir dirección en terreno, será nuevamente acordado este paso.

- Los integrantes del equipo, inicialmente, aportan desde los ámbitos profesionales propios hacia el tema que se aborda. Un equipo que realiza una propuesta territorial, aunque sea sectorial, está integrado por profesionales o estudiantes que abarcan dis- 
tintas áreas de conocimiento. Por ejemplo: en procesos ecosistémicos de espacios naturales, procesos productivos, abastecimiento y sistema urbano, gobernabilidad, comunicación, etc.

- Para llegar a la interdisciplinariedad comenzamos por crear el "campo común" de los miembros del equipo con las tendencias y pensamientos de los participantes y la forma de resolución de las diferencias frente a la acción.

- Durante el proceso de reflexión sobre el tema/objeto de atención se requieren análisis parciales sobre distintos temas confluyentes. Estos análisis se vuelcan posteriormente en la mesa de reflexión a fin de que sirvan de alimento y sean manejados por todos los integrantes.

- En el camino hacia la transformación de la mirada, sin duda, pueden aparecer "flujos de poder profesional" y "áreas inadvertidas", desde lo social, lo ambiental, lo productivo, lo proyectual, la planificación, etc. La advertencia de los contenidos implícitos que recorren los equipos, advertencia que casi siempre está a cargo del coordinador/facilitador, aunque estos contenidos pueden ser señalados por algún miembro, es planteada al equipo para que elabore o incorpore esos flujos o áreas que permanecen en la oscuridad e influyen en la tarea, tarea que consiste en arribar a momentos interdisciplinarios.

- Delimitar el alcance y la acción requerida al equipo de intervención es fundamental desde el comienzo, ya que un equipo que pierde el rumbo no se construye internamente. Puede modificar ese rumbo de acuerdo con lo que aparezca como contenido, aunque siempre tener presente la acción o meta a realizar

- Los equipos interdisciplinarios son equipos operativos, necesitan realizar la acción para la cual fueron convocados

- Durante y después de haber concluido la realización de la propuesta o proyecto, es fundamental una retroalimentación programada y continua y, posteriormente, un seguimiento más espaciado una vez instalada y en marcha.

Para esta etapa formativa en gabinete de la capacidad interdisciplinaria de un equipo, se propone una modalidad que incluye roles específicos de coordinación/facilitación, observación y registro.

Roles que serán cumplidos siguiendo los parámetros de los grupos operativos. Los grupos operativos tienen sus antecedentes establecidos en las investigaciones que llevan a cabo primeramente Kurt Lewin en el Massachusetts Institute of Technology (MIT), en los T Groups o grupos de diagnóstico, los grupos de creatividad y el Sensitivity Training, el socio análisis institucional y la corriente latinoamericana de grupos operativos que investiga y desarrolla los roles específicos que acompañan un proceso transformador. Al final de este artículo colocamos algunos títulos bibliográficos complementarios de estos antecedentes y los fenómenos investigados por estas corrientes.

En la Universidad del Nordeste Argentino se abre en los ochenta/noventa un espacio en Extensión Universitaria para el entrenamiento en coordinación, facilitación, observación y registro de grupos interdisciplinarios e intersectoriales. Esta práctica, sistematizada e investigada, aporta los elementos básicos teóricos para la formación en los roles y la participación grupal, complementada con un ciclo de encuentros teórico-prácticos-vivenciales propuestos por diferentes adalides de enfoques grupales. 
Para la formación en roles específicos grupales básicamente se requiere:

- Alrededor de 60 horas de práctica con el acento en coordinación.

- Investigación sobre el cumplimiento de esos roles.

- Diversidad de experiencias confluyentes en lo que se llama "laboratorio social", técnicas y metodología de aplicación en grupos interdisciplinarios.

- Reconocer y practicar los instrumentos de coordinación: señalamientos, reformulaciones, devoluciones, reconocimiento de los indicadores de poder, lectura de lo analógico grupal, clarificación de la información circulante. Método expansivo, asociación libre, prácticas convergentes, estrategias sociales de intervención e involucramiento.

- Práctica en la colaboración para construir el "campo común" del equipo, realizando las intervenciones y elaboraciones a eje centrado en el objeto de atención/intervención; y en abrir canales de escucha de los participantes del equipo hacia los aportes de todos los miembros y posteriormente hacia los aportes de los habitantes del lugar de intervención.

- Adquirir capacidad de detección y manejo de los conflictos grupales. Los grupos operativos transcurren por etapas, con elementos y situaciones observables, que es necesario traspasar a través del volcado al grupo, su reconocimiento y elaboración. Por lo tanto, es importante que coordinación conozca y reconozca esas etapas y elementos. Basándonos en un esquema simple, decimos que el proceso de los equipos hacia su transformación en equipos interdisciplinarios cumple, en general, tres estadios: inclusión, confrontación y organización para la producción. Cada uno de estos estadios muestra indicadores que son reconocibles y se pueden volcar al grupo. En la etapa inicial existen indicadores de conflictos relacionados a las ansiedades básicas. En la etapa propiamente dicha de confrontación, o conflicto grupal, se necesita disponibilidad y capacidad de escucha, ya que entra en juego la opción de seguir adelante y llegar a organizarse o tener dificultades y desmembrarse el equipo. Es imprescindible que los conflictos posibles salgan a la luz. Es en esta etapa de confrontación donde aparece el conflicto esencial frente a la tarea. La presencia de este conflicto esencial puede adquirir forma explícita o implícita. Si es implícita, la lectura de la comunicación no verbal del grupo puede indicar los signos para que coordinación trabaje en la resolución del mismo.

- Punto central es el volcado de la mirada al grupo como un todo recorrido por fenómenos que, analizados e incorporados a través de la palabra explícita, van constituyendo la amalgama que nos permite crear pensamiento sobre el tema o la acción que nos ocupa. En las etapas iniciales el grupo dedica su energía disponible a constituirse como tal, para comenzar a acceder, posteriormente, al enfrentamiento y resolución de la etapa de conflicto donde se libra la batalla sobre el enfoque esencial que lleva al pensamiento común.

El proceso formativo a través de roles específicos es un proceso de transferencia. No se trata de que estos roles específicos se instauren como un lugar de poder, sino, esencialmente, de instrumentalizar a los profesionales en formación para que participen en la construcción 
de los equipos. Profesionales que, una vez cumplido el ciclo formativo, deseen y necesiten aplicar esta construcción para la realización en un hábitat determinado.

Es probable que al conformarse los equipos para una intervención más allá del ámbito universitario, estos equipos se autogestionen o sus miembros cumplan rotativamente con roles de coordinación/facilitación. En este caso es importante tener en cuenta algunas reglas básicas para el funcionamiento autogestivo. Estas reglas se refieren básicamente a: aceptar los aportes de los miembros sin comenzar por negarlos; ubicarse en el presente; aportar por asociación sobre lo que se reflexiona; hablar en primera persona; no dirigirse hacia algún miembro en particular sino al grupo como un todo, etc. (Cá y Wainer, 1994).

Aunque hablamos en conjunto de coordinación/facilitación, ya que un coordinador también cumple con la función de facilitar, en sí la facilitación requiere menos entrenamiento que coordinación, ya que se facilita instrumentando para poder "escuchar" los aportes individuales a la trama que se va tejiendo, sin acaparar la palabra y aceptando la importancia de los aportes de todos y cada uno de los miembros.

El rol de coordinación en sí requiere no solo mayor entrenamiento sino también haber vivido esta práctica, ya que este rol trabaja sobre los contenidos implícitos y la decodificación de los mismos es fundamental para la marcha del grupo, especialmente aquellos contenidos que señalan la aparición de los flujos de poder (Lucca, 1995; Lucca, 2014, p. 33).

\section{El proceso interdisciplinario y los procedimientos metodológicos}

El camino o proceso hacia la interdisciplina implica varios momentos que es importante visualizar y que son vitales para la creación de la cancha reflexiva interdisciplinaria. Momentos que han sido investigados y consignados de la siguiente manera (Lucca, 2014):

- 1er. momento: fuerte relación propia disciplinar. Intervenciones desde el lugar de la propia disciplina. Incapacidad de "escuchar" los aportes de otras disciplinas.

- 2do. momento: con la aparición de cierta interrelación afectiva (de respeto y aceptación) entre los miembros, despuntan momentos de "apertura" de la escucha a lo que dice el otro. A medida que esta apertura de "escucha" se va agrandando surgen las primeras crisis al ponerse en juego, cuestionando o no, los propios pensamientos, conocimientos o sentimientos sobre los temas tratados, aunque esto significa continuar referenciándose al propio conocimiento.

- 3er. momento: primera crisis epistemológica generalizada. Se cuestionan, interna y externamente, los saberes, y aparece la pérdida de seguridad. Es un momento particular donde emerge la duda frente al conocimiento adquirido. Es necesario reforzar el camino para no desear volver hacia atrás.

- 4to. momento: sobreviene confusión sobre cómo encarar las situaciones/temas/objeto de intervención del grupo interdisciplinario. Y se enuncia una de las reglas de la interdisciplina: el "principio del silencio ante la pregunta frontal".

- 5to. momento: se comienza a practicar formas de tejer la visión sobre el objeto desde los distintos ángulos, observando la deformación que este objeto adquiere a partir de las distintas miradas. 
- 6to. momento: se entrega el dominio de la comprensión del objeto a la revisión conjunta. Es el primer momento interdisciplinar.

- 7mo. momento: el ejercicio de una mirada multifocal permite ampliar la conciencia incorporando una visión organizada, concertada, transdisciplinar.

Este proceso o camino para adquirir las formas de reflexión interdisciplinar es recorrido una y otra vez por los grupos en situación de crear una propuesta. Lo importante es que una vez practicado se adquiere confianza en esos momentos de quiebra y pérdida de seguridad.

El proceso seguido por el grupo no es ciertamente lineal. Para llegar al momento interdisciplinario fundante, se vuelve repetidas veces a los momentos $1 .^{\circ}$ y $2 .^{\circ}$. El momento $3 .^{\circ}$ se aborda en tres ocasiones, pivoteando entre el $5 .^{\circ}$ y el $3 .^{\circ}$, hasta que finalmente se accede al $6 .^{\circ}$ momento, para luego llegar al $7 .^{\circ}$ en la creación del plan. La totalidad del proceso se desarrolla en tres meses (Lucca, 2014, p. 31).

La interdisciplina es un camino de reconstrucción después de la deconstrucción del conocimiento unidisciplinar, una reconstrucción necesaria. Por lo tanto, no es un camino fácil, sino un proceso a seguir que se incorpora en nosotros como experiencia vital.

Para seguir este proceso proponemos algunos procedimientos metodológicos:

La creación del campo común. Crear la matriz grupal consensuando conceptos, terminología, enfoques. Esta matriz común nos permite converger en nuestras ideas e instaurar, desde cada disciplina, aportes con distintos niveles de protagonismo y homogeneidad, es decir, no primando un solo enfoque disciplinar, sino determinando las ideas consensuadas que van a protagonizar la propuesta.

El método expansivo a través de un proceso de asociación nos permite buscar los contenidos que no son claramente explícitos, pero que cuentan en las decisiones y nos involucran vitalmente. Contenidos latentes que por asociación sugieren nuevas formas de encarar los puntos a los que va arribando el equipo. Es tarea de coordinación no perder de vista la mirada convergente hacia el objeto/situación de atención del trabajo. El método expansivo, sin embargo, puede hacer surgir esos elementos serendipíticos que permiten ir más allá.

$\mathrm{Al}$ emplear estos procedimientos es probable que nos descentremos de las disciplinas propias y respondamos a las necesidades vitales y no a las respuestas establecidas de las referencias disciplinares. Este descentramiento nos permite volvernos creativos y encontrar soluciones no estereotipadas ni influidas por las estructuras políticas del "hacer" profesional.

La metodología de la intersubjetividad objetiva implica liberar los propios contenidos y al mismo tiempo consensuarlos con los otros miembros. El hecho de consensuar lo que subjetivamente pensamos o visualizamos lleva a una objetivación del tema que nos ocupa, necesaria para definirla y poder operar sobre ella.

Toda disciplina nos instrumentaliza, aunque al mismo tiempo nos sujeta a respuestas ya conocidas y por lo general centradas en la lógica establecida de esa disciplina. La interdisciplina requiere e incita a traspasar esos límites para conformar respuestas que, por sus características de contener diversidad de enfoques, pueden ajustarse a una realidad más amplia y más contenedora. 
Por otra parte, sabemos que las formaciones disciplinares tienen un sesgo político académico que, por satisfacer el estado estructural imperante, responde a un esquema de conocimiento determinado, una lógica de mercado, etc. Este esquema de conocimiento puede alejarse, salvo casos especiales, de lo que las realidades complejas del hábitat demandan.

Para aplicar las propuestas que surgen de un equipo que las ha trabajado interdisciplinariamente, es primordial el encuentro con los sujetos o grupos que van a convivir con estas propuestas. Y en ese encuentro aparecen las estrategias de aplicación o intervención necesarias, ya que es con los propios habitantes del territorio a intervenir con quienes establecemos estas estrategias de intervención en un involucramiento progresivo. Dicho de otro modo, cuando estamos en situación de establecer estrategias de intervención, es el momento de incluir a los habitantes en nuestras propuestas e ir a terreno.

Si lo hiciéramos antes de que el equipo hubiera realizado el proceso antes descrito, los habitantes encontrarían un conjunto de profesionales en el que cada uno desea convencer de su propio enfoque, lo cual crea una respuesta no adecuada.

\section{Hábitat, equipos interdisciplinarios y habitantes de un territorio}

Cuando hablamos de hábitat nos referimos a "los procesos que tienen lugar en un sistema complejo y que explican su comportamiento y evolución como totalidad organizada" (García, 2011, p. 67). En la Figura 1, un grupo integrado por profesionales, intendentes y autoridades de las comunas del Adriático, norte de Italia, y vecinos de las mismas, revisamos
Figura 1. Análisis del territorio para la planificación de la sustentabilidad en cinco comunas sobre el mar Adriático, Italia. Fuente: Registro de la autora.

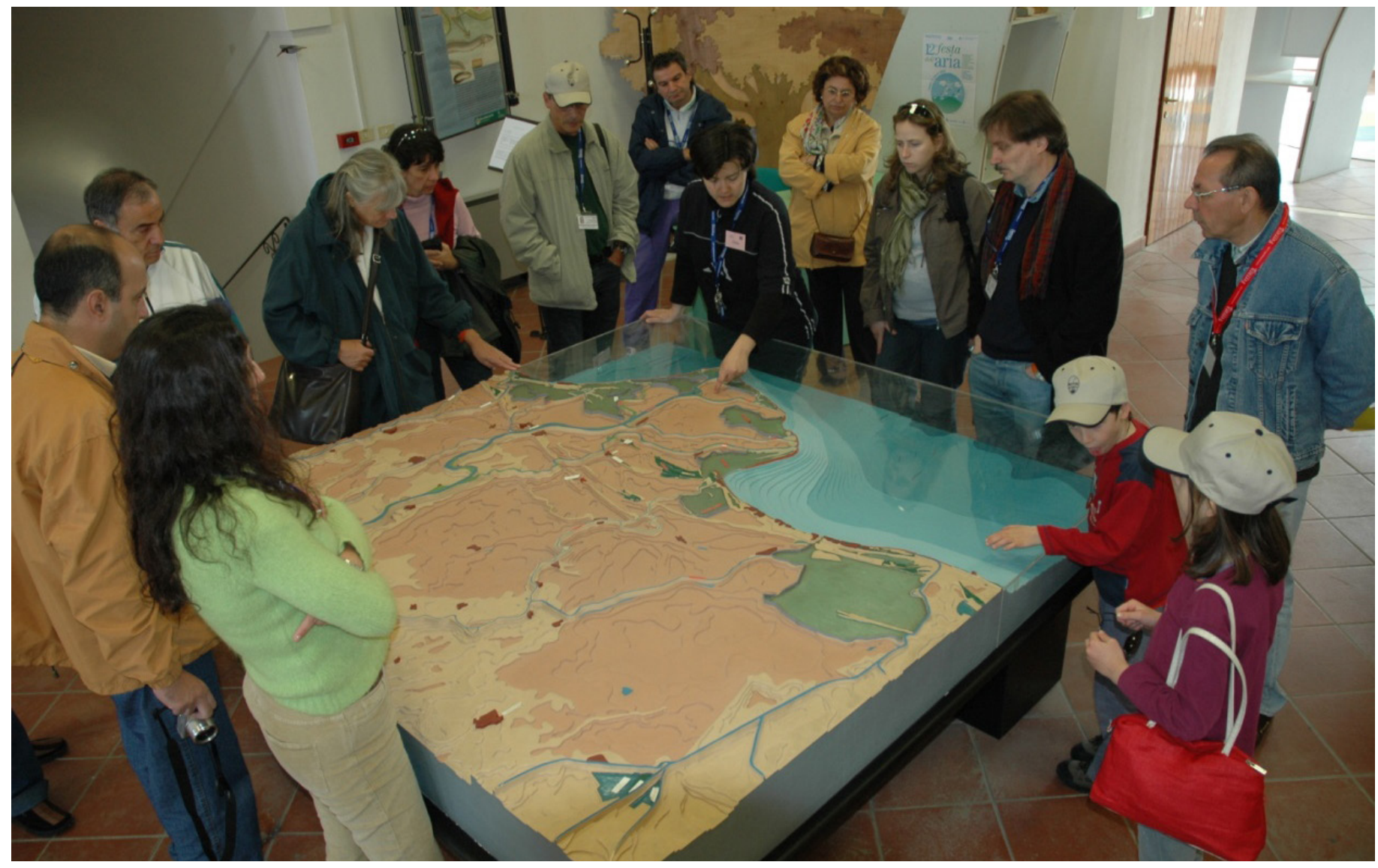


los procesos de la zona antes de tomar contacto directo con el territorio. Como objeto de estudio y de operatividad, nos referimos genéricamente al espacio geográfico, o conocimiento objetivo del territorio $(\mathrm{T})$, la dimensión subjetiva de los lugares o territorialidad (Te) y la gobernabilidad de ese espacio que da cuenta de las continuas transformaciones o territorialización (T') (Ferrier, 1998).

Desde la concepción de la sustentabilidad, el hábitat está integrado por espacios naturales, urbanos y rurales, siendo la proporción entre estas tres dimensiones un tema importante a tener en cuenta, ya que el espacio natural es el que sustenta los otros dos (Lucca, 2016). Significa que cuando hablamos de hábitat pensamos en un espacio a planificar con sus características de habitabilidad y las interdependencias e interrelaciones de estas tres dimensiones, la natural, la urbana y la rural.

Por lo general, se relaciona espacio urbano con habitantes, usuarios de ese espacio. Y se da por hecho o se habla de la importancia del espacio natural "para recreación" de los humanos. Esta es una concepción que es necesario rotar dándole el protagonismo al espacio natural donde vamos a instalarnos, ya que de esta rotación dependen los beneficios o problemas que tendremos posteriormente en nuestros asentamientos. En el encuentro de los equipos interdisciplinarios con los habitantes del lugar, esta rotación de mirada hacia lo natural, que se desplaza de lo construido hacia el territorio natural existente, es fundamental. Y es importante instalarla desde el comienzo de una relación de intervención.

Consideramos que los habitantes de un espacio geográfico son los conocedores de su territorio, y son ellos, con sus gestos y decisiones, los que accionan y conviven en él. El encuentro con ellos marca un inicio, un desarrollo y un acompañamiento para la concreción de una acción. Y desde el primer momento el tema es involucrarnos en conjunto, transferir e instrumentar para producir esa acción.

Por lo general las estrategias iniciales que involucran a los habitantes son aquellas que les permiten expresar sus intercambios y sentimientos con respecto al lugar que habitan o que desean habitar. Más allá de que ellos hayan solicitado una intervención o no, el hecho de que se reconozca sus conocimientos y sentimientos sobre su lugar, y que el equipo pueda escuchar e interpretar esos conocimientos, significa que se va construyendo una identidad de pensamiento común sobre el espacio-hábitat. Un equipo interdisciplinario cumple un papel de intermediario entre los habitantes, el espacio en sí y las instituciones que intervienen en ese espacio. Rol de intermediario que ha sido objeto de capacitación en el proceso de formación interdisciplinario.

Tanto si la necesidad de la propuesta parte de una institución en particular como de la detección de la misma por parte del equipo, o de los pobladores, es fundamental concertar con los habitantes o posibles habitantes de ese hábitat. Concertación necesaria para que los habitantes se apropien de la propuesta y comiencen a ser activos participantes, corporizándose en ellos la propuesta al confrontarla con el equipo hasta definir formas comunes de acción.

Desde nuestro punto de vista, es indispensable este encuentro inicial de conocimientos mutuos entre habitantes y equipo. Y para que este paso sea una alianza estratégica con los territorios, desde el comienzo introducimos la presencia de ese territorio en la relación.

Hay varias maneras de abordar la presencia del territorio. Particularmente, en distintas oportunidades, lugares y situaciones, hemos aplicado algunas de las formas que mencionamos acá. Una de ellas es 
comenzar valorando la presencia de lo que llamamos los servicios ecosistémicos del medio natural, como esencial para la habitabilidad del espacio.

\section{Servicios ecosistémicos}

Capital natural $=>$ flujo de servicios $=>$ bienestar humano

Los servicios ecosistémicos se refieren: a) a lo que nos provee el lugar, alimentos, materias primas, agua, recursos medicinales, etc.; b) a la regulación del clima local, del secuestro de carbono, eventos extremos, tratamiento de efluentes, control de erosión, regulación de la polinización, del control biológico; c) hábitat para especies, para la diversidad genética; d) recreación, salud, turismo, inspiración estética.

Otra manera de valorar los espacios con los futuros o actuales habitantes es proponerles una expresión de los valores de uso, directo e indirecto, y los valores de no-uso. Esquematizamos (Guadro 1):

\begin{tabular}{|c|c|c|}
\hline Valor de uso & Valor intangible & \\
\hline Directo & Indirecto & Existencia/Cultural \\
\hline $\begin{array}{l}\text { Plantas y animales no cultivados ni } \\
\text { domésticos que se consumen o ven- } \\
\text { den: } \\
\text { - Comida } \\
\text { - Medicinas } \\
\text { · Materiales } \\
\text { Construcción } \\
\text { Recursos o beneficios directos que } \\
\text { no se consumen: } \\
\text { - Sombra de árboles } \\
\text { - Uso de bosques cementerios } \\
\text { - Uso de especies salvajes para mejo- } \\
\text { rar especies domésticas }\end{array}$ & $\begin{array}{l}\text { Especies o sistemas que soportan } \\
\text { otras actividades económicas: } \\
\text { - Rol de áreas boscosas para proteger } \\
\text { cauces de ríos o cuencas y regulación } \\
\text { de inundaciones } \\
\text { - Ciclo de nutrición en tierras de culti- } \\
\text { vo promovidas por sectores naturales } \\
\text { - Polinización provista por especies } \\
\text { de pájaros, abejas }\end{array}$ & $\begin{array}{l}\text { Especies o sistemas valorados por sus } \\
\text { derechos propios sin referencia a un } \\
\text { uso económico: } \\
\text { - Importancia cultural y herencia pa- } \\
\text { trimonial } \\
\text { - Belleza y paisaje } \\
\text { - Motivación para preservar recursos } \\
\text { para futuras generaciones (incluyen } \\
\text { una amplia gama de recursos de la } \\
\text { diversidad biológica) }\end{array}$ \\
\hline
\end{tabular}

Tanto el valor de uso como los valores culturales intangibles, que los humanos determinamos para algunos lugares, requieren ser tenidos en cuenta, ya que identifican a algunos pueblos o sociedades. Por ejemplo, en una isla del río Paraná en el sur de Latinoamérica, los pobladores rescatan los sonidos de ese lugar, producidos por el viento, el río deslizándose, la vegetación costera, los monos que habitan allí y las especies de aves; sensaciones que, según ellos, logran que los habitantes humanos gocen de actitudes no agresivas y un trato amable. En un libro de mi autoría desarrollo el caso piloto de aplicación en un territorio, isla del Cerrito de la provincia del Chaco, Argentina, donde sus pobladores realizan un proceso para reconocer y valorar las riquezas de su propio territorio, ignoradas hasta el momento, como ellos mismos expresan, entre ellas los sonidos del lugar (Lucca, 2016; González Cruz, 2016). Otra de las formas es comenzar evaluando el estado de los elementos soporte del ambiente: el estado del agua, del suelo, del aire, de la cobertura verde del espacio.

Trabajamos con estos enfoques en barrios de la ciudad de Barranqueras, provincia del Chaco, Argentina; barrios ubicados alrededor de lagunas pertenecientes a los humedales correspondientes al río Paraná; en ciudad de Fontana, también en territorio de lagunas; en Machagai, población del interior de la provincia del Chaco, con un historial de pérdida de cobertura boscosa y una población dedicada a la produc-
Cuadro 1. Valoración de los espacios habitables. Fuente: Elaboración propia. 
ción primaria forestal; en Clorinda, pueblo de la provincia de Formosa, Argentina, con una problemática de contaminación del río Pilcomayo por descarga de residuos urbanos; en provincia de Corrientes, Ituzaingó, con barrios que presentaban degradación de construcción urbana y problemas sociales; y en barrios de las ciudades de Resistencia y Corrientes de Argentina, nuevos asentamientos o barrios construidos por el organismo oficial de vivienda.

Estas propuestas han comenzado con la aplicación de la metodología que llamo PEA, Percepción de los Espacios Ambientales naturales, urbanos, rurales, de simple manejo y observación para los habitantes de esos espacios. Una metodología que propone un proceso que implica etapas: inicio, desarrollo, planificación, ejecución y monitoreo. Y que utiliza diversos procedimientos o instrumentos para transferir formas y contenidos sobre distintos temas a estudiar en cada lugar. Uno de ellos, el trabajo con indicadores. Construimos, con los habitantes de las poblaciones más el equipo técnico interdisciplinario, los ICP, Indicadores de Captación Perceptiva, adecuados a cada territorio y que muestran el estado de los elementos base del ambiente señalando las vías de recuperación de los mismos y las relaciones con lo construido. Estos indicadores nos señalan las acciones necesarias, simples y cotidianas a cargo de pobladores, o más complejas, en las que se involucra a los organismos reguladores. Por ejemplo, la selección y procesamiento domiciliario de residuos para evitar la descarga a los espejos de agua, regular el sistema de descarga de aguas marrones, etc.

Hemos utilizado, además: el "mapeo" de producción y de redes sociales, como punto de partida de las observaciones; las caminatas o recorridos silenciosos; la metodología REFLECT basada en Paulo Freire; el DRP, Diagnóstico Rápido Participativo; la PS, Percepción Sensorial; la graficación de los flujos de circulación genética; el TPD, Teatro para el Desarrollo; etc. Para la utilización de estos procedimientos sugerimos que algunos miembros del equipo interdisciplinario o el equipo completo los haya experimentado en su proceso de capacitación.

Valorar el capital natural existente es una tarea ineludible del hábitat (Sarmiento, 2016). Y medir su estado de salud o degradación es tarea básica, tanto antes de ubicar un asentamiento humano o sobre uno establecido. Cuando este capital natural requiere reconstitución, los indicadores nos describen su comportamiento actual que, en relación al ideal, nos permite pensar las acciones a realizar. Si nos encontramos ante un espacio carente de cobertura, lo deseable sería permitir su reconstitución o planificarla e iniciarla antes de ubicar el asentamiento humano. Un ejemplo simple: existe un programa francés que propone un preverdissement, poblar de cobertura los espacios que ya no la poseen, antes de construir sobre ellos.

El territorio, la territorialidad y la confrontación con la territorialización son los núcleos de nuestro encuentro y reconocimiento en terreno. El territorio implica la estructura física objetiva del espacio que abordamos. Mientras, la territorialidad es la forma subjetiva como los humanos vivimos y sentimos los lugares con sus características típicas, en los que habitamos o transcurrimos. Con respecto a la territorialización -los planes, programas, proyectos y acciones propuestas por autoridades e instituciones-, la evaluación conjunta de lo que se propone en el territorio aporta indicaciones concretas y modificaciones a partir de la conceptualización del metabolismo del territorio, que se realiza en el 
encuentro entre pobladores, equipo y autoridades. En el ejemplo anteriormente citado de la isla del Cerrito, el municipio del lugar modificó su gestión de los espacios públicos de la isla, playa, camping, alquiler de tierras para pastoreo, etc., implicando un ahorro financiero significativo y un ordenamiento administrativo.

La reflexión e investigación sobre cómo recuperar la salud de los espacios e instalarse en ellos instaura formas de acción que resultan en un aprendizaje tanto para las instituciones como para los equipos y habitantes que van a convivir con esos espacios. Repensar estos puntos a partir de recorridos, análisis, búsquedas de asesoramiento sobre los comportamientos del territorio y una recuperación de la territorialidad con los habitantes nos dan el material para comenzar un plan de acción que integre espacio natural con objetos, infraestructura, viviendas, servicios, etc. Esta base lleva a encarar los elementos que introduzcamos, evaluando sus características más o menos invasivas o/y contaminantes, ya que nos guiamos teniendo como norma la existencia o la recuperación de los beneficios ambientales de los espacios que abordamos.

El trabajo inicial, con el surgimiento del protagonismo de los pobladores, es la etapa fundante, a la que seguirá la elaboración hasta la visualización completa del espacio y sus componentes, para pasar a la enunciación de un plan con responsables, a partir del cual se inicia la ejecución del mismo.

El desarrollo de toda propuesta involucra a distintos actores, aunque tanto equipo interdisciplinario como población llevan una relación de compenetración frente a la creación que están instaurando. Esta compenetración de equipo/habitantes puede ser fructífera y aprendizaje de habitabilidad para ambos, así como fuente de permanente retroalimentación.

Los mecanismos de toma de decisiones en esta relación van más allá de la búsqueda de consenso. El equipo trae ya establecido su mecanismo para enunciar ideas interdisciplinarias, y al construir con los pobladores un intercambio comienza a aparecer una "mirada común" que definirá una propuesta. A partir de allí se van a evaluar posibles acciones, diseñadas entre actores, que conllevan insertar la información inicial aportada por el equipo interdisciplinario. Esta elaboración conjunta es creativa y desemboca en propuestas de las cuales pueden todos hacerse cargo y fundamentar.

También es importante mencionar que, al elaborar una revisión compleja sobre los temas del territorio, se llega a puntos críticos al descubrir que ciertas acciones cotidianas van en contra de nuestro propio bienestar y de la salud del lugar donde vivimos, demandando un cambio. Enfrentar estos momentos y temas críticos, y elaborarlos para producir el cambio necesario, implica la capacitación en interdisciplinar del equipo y la transferencia de esta capacitación al trabajo conjunto.

Cada lugar, cada conjunto de pobladores, requiere distinto tipo de aproximaciones. Casi siempre lo que ancla profundamente en las personas, en las sociedades, es el reconocimiento de sus deseos y sensaciones que pueden servir de base para la elaboración de los cambios en la mirada sobre su territorio. Cambios con una direccionalidad hacia un territorio que sea proveedor y contenedor.

Conjugar con la realidad del territorio al que nos aproximamos es nuestra tarea y desafío, construyendo a partir de allí, sin soluciones fijas de antemano, un camino de aprendizaje común. 


\section{Conclusiones generales}

Consideramos la universidad como un ámbito propicio para la capacitación en interdisciplina, ya que por su función formativa posee los recursos humanos que requiere esta capacitación, los espacios interfacultades para desarrollarla y la posibilidad de establecer formas de complementariedad de los conocimientos disciplinarios que allí se imparten, en una etapa simultánea a la adquisición de los mismos, así como campos de práctica para desarrollarla. Por otra parte, producir este capital social, como podríamos nominar a los equipos que se han formado interdisciplinariamente, es función de una institución con autonomía posible en cuanto a concepción política/económica/social/ambiental/cultural.

En el desarrollo de este documento argumentamos que la formación interdisciplinaria se constituye a través de un proceso que es imprescindible transcurrir. Proceso que requiere ser conducido para su aprendizaje, aportando aquí algunos elementos para la conducción y el enfoque de esta formación.

Llegar a los momentos interdisciplinarios significa producir un cambio en la forma de enfocar los temas que requieren atención profesional. Este cambio produce una ruptura del enfoque unidisciplinar al incluir los enfoques de las otras disciplinas comprometidas en acciones complejas, para llegar a una mirada unificada, objetivo interdisciplinar.

La interdisciplina aplicada al hábitat implica llegar a la comprehensión de una habitabilidad saludable, por lo tanto sustentable. La acción en hábitat se presenta como un complejo de situaciones que es necesario aprender a descifrar teniendo en cuenta todas sus características. Acción que es necesario repensar y componer desde distintos ángulos que nos permitan crear una forma diferente a la habitual y que, al mismo tiempo, rescate todos aquellos conocimientos adquiridos que se suman a los establecidos en nosotros desde nuestra capacidad como organismos captores de signos vitales, signos que existen en los fenómenos que nos rodean y podemos registrarlos como indicadores de esa habitabilidad saludable.

Cuando los equipos ya constituidos van a terreno, toman contacto con un hábitat constituido por los elementos y especies que habitan en ese espacio, que incluye o va a incluir a la especie humana, y que demanda un replanteo en la forma de habitar. Replanteo que los equipos interdisciplinarios pueden colaborar a construir, e instar a los sujetos habitantes también a hacerlo. Para dar este paso tenemos en cuenta establecer una relación de retroalimentación mutua de pobladores y equipo, poniendo el acento en el protagonismo de los pobladores del lugar. Y además volcar la atención desde el comienzo, hacia el espacio a trabajar en todas sus dimensiones, la natural, la urbana o construida, y si es posible la rural.

Proponemos mirar el territorio y sus características, recuperar la territorialidad y valorizarla, y a partir allí proponer formas de territorialización. Decimos que para que las formas de habitar humano comiencen a tender a la inclusión del espacio donde se asientan, ese equipo, primeramente en sí, desarrolla un pensamiento que va entrelazando y tejiendo desde las distintas áreas de conocimiento, practicando la inclusión de diversidad desde la escucha y la conformación de una mirada común hacia un territorio que requiere direccionarse hacia lo sustentable, para luego transferir formas de pensar y mecanismos de decisión en común a los pobladores, con quienes se realiza un reconocimiento del lugar, base de las acciones posteriores. 
Aprender a habitar de manera inclusiva, y no hablamos solamente del aspecto social, es el resultado de estos procesos. Además, realizar la transferencia y conjugar con los habitantes que permanecerán en el lugar, es construir la capacidad de estos para la continuación de la vitalidad de sus espacios en el futuro. Esta vitalidad asentada en el aprendizaje de la convivencia con lo existente. 
Cá, K. y Wainer, A. (1994). Grupos de Autogestión. Buenos Aires: Ed. Era Naciente.

Carreño. F. et al. (2003). Planeación del Territorio y Ambiente en América Latina. México: Selección del CIUMA III Congreso Internacional de Urbanismo y Medio Ambiente. Ed. Facultad de Planeación Urbana y Regional, UNAM Universidad Autónoma del Estado de México. Tomo I y II.

Ferrier, J-P. (1998). Le Contrat Géographique, ou l'habitation durable des territoires. France: Editions Payot, Lausanne.

García, R. (2011). Interdisciplinariedad y Sistemas Complejos. Revista Latinoamericana de Metodología de las Ciencias Sociales - ReLMeCS, 1 (1). Recuperado el 10 de mayo de 2017 de: http:/ / contenidosabiertos.academica.mx/jspui/bitstream/987654321/504/1/interdisciplinariedad_y_ sistemas_complejos.pdf.

González Cruz, F. (2016). Identidad y Desarrollo Local. Chile, Santiago. Revista Sustentabilidad(es), 7 (14), 27-47. Recuperado el 10 de mayo de 2017 de: http://www.sustentabilidades.usach.cl/sites/ sustentable/files/paginas/gonzalez_francisco._ identidad_y_desarrollo_local.pdf.

Lucca, E. (2002). Desarrollo Sustentable, Conflictos Ambientales y Paz. España, Barcelona. Agathos, 2 (2).

- (2006). Decidir el Futuro. La participación, gestión para la sustentabilidad ambiental y Agenda Local 21. Resistencia: Ed. Arbres de Vie, Librería de la Paz.

- (2010). Sustentabilidad urbana, rural, natural. Chile, Santiago. Sustentabilidad(es), 2 (8). Recuperado el 10 de mayo de 2017: de http://www.sustentabilidades.usach.cl/sites/sustentable/files/paginas/02-08.pdf.

- (2014). Interdisciplina. Práctica esencial para el abordaje social y ambiental. Resistencia: Ediciones Arbres de Vie, Cospel.

- (2016). La Gestión de los Territorios. La Cosecha Escondida o la Percepción Ambiental de los Espacios. Buenos Aires: Ed. Diseño.

Peredo Parada, S. y Aedo Zúñiga, M.P. (2016). Complejidad y multidisciplinariedad en el aprendizaje de la sustentabilidad: la experiencia del Diplomado en Educación para el Desarrollo Sustentable de la Universidad de Santiago de Chile. Sustentabilidad(es), 7 (14), 76-87. Recuperado el
10 de mayo de 2017 de: http:/ /www.sustentabilidades.usach.cl/sites/sustentable/files/paginas / peredo_aedo._complejidad_y_multidisciplinariedad_en_el_aprendizaje_de_la_sustentabilidad._la_ experiencia_del_diplomado_en_educacion_para_ el_desarrollo_sustentable.pdf.

Primer Congreso de la Transdisciplinariedad (1994). Carta de la Transdisciplinariedad, Portugal. Convento de Arrábida. Recuperado el 10 de mayo de 2017 de: http:/ / www.filosofia.org/cod/c1994tra.htm.

Rieznik Lamana N. y Hernández Aja A. (2005). Huella Ecológica, España, Madrid. Ciudades para un Futuro más Sostenible. Recuperado el 10 de mayo de 2017 de: http:/ / habitat.aq.upm.es/temas/a-huella-ecologica.html.

Sarmiento, L. (2016). Bioética Urbana al cuidado de la vitalidad colectiva de los territorios. Sustentabilidad(es), 7 (14), 88-106. Recuperado el 10 de mayo de 2017 de: http:/ / www.sustentabilidades. usach.cl/sites/sustentable/files/paginas/sarmiento_laura._bioetica_urbana_al_cuidado_de_la_vitalidad_colectiva_de_los_territorios.pdf.

\section{Referencias complementarias}

Amado, G. y Guittet, A. (1975). La dynamique des communications dans les groupes. France: Ed. Librairie Armand Colin.

Lapassade, G. (1985). Grupos, Organizaciones e Instituciones. Barcelona: Ed. Gedisa.

Egan, G. (1976). El Laboratorio de Relaciones Interpersonales. Teoría y Práctica del Sensitivity Training. Buenos Aires: Ed. Paidós.

Knapp, M. (1980). La Comunicacion no verbal. El Cuerpo y el Entorno. Buenos Aires: Ed. Paidós.

Lucca, E. (1995). Buscando la sinergia en el trabajo social. Pensamientos sobre el eros grupal. Buenos Aires: Ed. Humanitas.

Lobrot, M. (1994). Pedagogía Institucional. Buenos Aires: Ed. Humanitas.

Mendel, G. (1972), Sociopsicoanálisis 1. Buenos Aires: Ed. Payot-Amorrortu.

- (1973). Sociopsicoanálisis 2. Buenos Aires: Ed. PayotAmorrortu.

Pichón-Rivière, E. (1975). El Proceso Grupal. Buenos Aires: Ed. Nueva Visión. 
Lucca, E. (2017). Estrategias y metodologías de formación de equipos interdisciplinarios para trabajar en hábitat. Hábitat y Sociedad, 10, 15-34.

<http://dx.doi.org/10.12795/HabitatySociedad.2017.i10.02>

$\bigcirc$ 


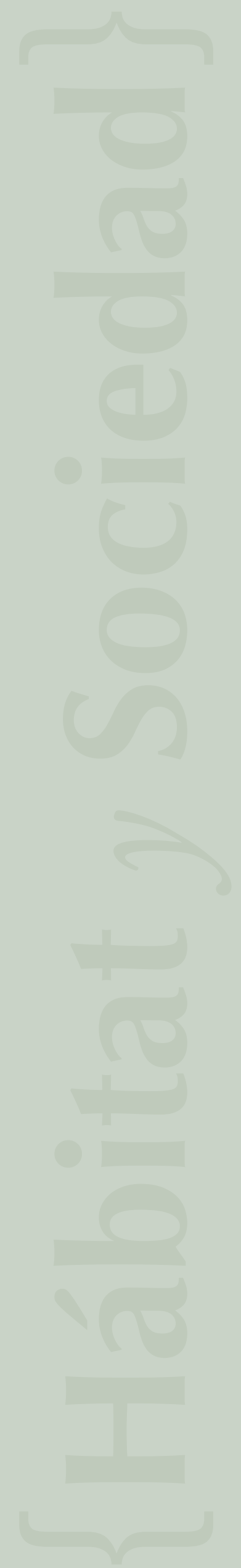

